\title{
Distributed and Specialized Agent Communities
}

\author{
Jesús Ángel Román, Sara Rodríguez, and Juan Manuel Corchado
}

\begin{abstract}
SCODA (Distributed and Specialized Agent COmmunities) is presented, like a new modular architecture for multi-agent systems development. By means of SCODA, multi-agent systems development is allowed under specialized modular philosophy, through it, the functionalities of the system can be extended in scaled form, according to the objectives. SCODA is composed by small subsystems of agents called, Specialized Intelligent Communities (CIE), which provide the necessary functionalities to solve the objectives needed across distributed services. By means of these CIE, scalability of the systems is allowed, so that they could be re-used in different developments, independently of his purpose.
\end{abstract}

Keywords: multi-agent systems, virtual organizations, dynamic architectures, specialization.

\section{Introduction}

The development of increasingly complex systems entails the need for develop capable components to be reused, so that the functionality that they provide can be used in other developments, keeping a compatibility between them. This philosophy is one that follows the object-oriented technology [1][2], where objects are encapsulated independently and can be reused in different developments with very different purposes, giving developers a significant advantage in terms of time spent in developing the system. This approach focused on Multi-agent Systems (Multi-Agent Systems, MAS) involves that, the development of an application based on multi-agent systems can be used in other developments, even with the overall purpose very different. For carrying out this approach, we need think that the set of agents that form a multi-agent system has to keep certain standardization and size, to be reused.

Jesús Ángel Román · Sara Rodríguez · Juan Manuel Corchado

University of Salamanca, Salamanca, Spain

e-mail: $\{$ zjarg, srg, corchado\} @usal.es 
The concept of organization has been studied extensively in sciences such as economics, sociology and psychology, and have also been several authors who have applied the concept of organization to development of multi-agent systems [3][4][5]. Applying the concept of organizing on multi-agent systems further increases the efficiency of itself having in account there is a check on the objectives to be met, both individual and collective, are established norms of behavior among agents, somehow there is a tasks division or specialization, that is, the function of the system is normed [6][7][8][9][5] If it also wants that a multi-agent system has the ability to be used in different developments regardless of their overall purpose, it would be necessary to define a new modular architecture and specialized, based on some sort of minimum size organization that establishes coordination norms between agents. The architecture would have a high degree of specialization at the organizational level, because the architecture of a multi-agent system determines the composition of the system itself, and the mechanisms used by agents to interact with their environment [10].

The main idea is design and implement a standard multi-agent architecture that meets the necessity of be reused in several developments in an efficient way. This architecture is based on a philosophy of business organization to improve the integration and development of multi-agent systems, where it is improved the distribution of the agents involved in the system and the services they implement. For this reason, is introduced the concept of community of agents as an organizational unit, and the concept of specialization applied thereto in order to obtain an optimization in the management and implementation of the system to be developed.

The article is structured as follows: Section 2 introduce several concepts, important for this work. Section 3 introduces SCODA, the architecture developed in this work. Finally, some experimental results and conclusions are given in Section 4 and 5.

\section{Related Work}

A multi-agent system is composed by a group of agents which act as some kind of organization. The main properties to characterize the internal behavior of the agents are: the type of reasoning and how they act, for example, reactive, modelbased, based on goals. Its adaptability, its perception and characterization the environment in which they are situated, including its computer infrastructure and their relationships with other agents, and the degree of autonomy in the actions they take [29]. The organization is used to describe this group of agents that are coordinated through a series of patterns and the establishment of some roles to achieve the objectives of the system. There are various approaches that exist in this area [6][11][4][7][8], depending on the structure and internal organization of the organizational structure. The behavioral, the objectives and the interaction of the agents are features that mark its global behavior and the aims. 
The concept of community differs from concept of organization that is more spontaneous and natural. The community relies on the common interests of those who work in a facility, and to carry out its regulation requires a system of comanagement contract [12][13] [14] In the work of [15][16] the term community is used to define a group of heterogeneous agents. Another agent community approach is given in [11], which defines the structure of the Cougaar agents.

This paper presents the definition of Intelligent Communities (CI). These communities, adopt features defined in other organizations of agents besides the features defined in Cougaar. Intelligent Communities are composed of a small number of agents, so that the communication between the agents is multidirectional, and not entail added computational effort. Decision making is centralized in decisions relative of whole community and will be independent for each agent in the work they perform individually, always to the benefit of the community. For this there is a formalization of the tasks thus providing a tool for agents for individual decision making. In an Intelligent Community there will be a two-level hierarchy as to the work of community control. One agent will be involved in the control over the work of other members having account as based a series of community rules that ensure the proper functioning of the same. This is because, although the members of the community are presupposed benevolent, in a community of agents, we have to take into account individual goals and deviations that may occur on the overall goals of the community.

Something remarkable is that, the Intelligent Communities must possess the ability to function in a distributed way at the community level and the services they provide should not be embedded in the members of it, but is necessary they are implemented in a distributed way, with the propose to free the community structure, and the member agents, of computationally load.

Specialization is a feature that gives to an individual belonging to a group, a peculiarity within that group. This specialization is observed within organizations as a means to achieve greater efficiency in the objectives pursued by it. The specialization, in the field of agents has been discussed in depth by [17][18][19] among others. This specialization aims to provide a multi-agent system more efficient, so that there is an improvement in the achievement of its overall objectives. One aspect to consider is the type of specialization needed that adapts to a group or organization. This typology must implement improvements in processes and objectives.

We define the specialization of tasks as the number of different tasks to develop in a working place, and how often they are repeated, and the power of decision exercised by the working place over the design of itself. The specialization of tasks, in turn, is divided into horizontal and vertical specialization of tasks [20]. The relationship between these two types of specialization is too narrow, since, a job in which there is a high horizontal specialization also requires a high vertical specialization, because the person performing a given task can lose the overview of objectives, and therefore it is necessary that another person, who has the vision, plan, organize and control the work [20]. 
The organizative specialization approach can be seen as a unit, across enterprise networks. In literature, the concept of enterprise network is found in the works of authors cited in [22], for which, a set of groups, institutions or organizations can interact with each other, getting favorable results, as much individual units in the network, as a whole. Thus, a corporate network can be considered as an association of companies working together so that there is a specialized complementation between them, with the aim of resolving situations which could not resolve individually, in the most efficient way possible.

\section{SCODA}

SCODA (Distributed and Specialized Agent Communities) [21] is a new architecture that focuses on the development of multi-agent systems. It is based on five principles: standard, specialization, ease of implementation, reuse and distributed computing. SCODA integrates one or more Intelligent Communities provided specialization, which we call Specialized Intelligent Communities (CIE) [21]. These CIE have the ability to function as an multi-agent system, independent and specialized, where the services offered are implemented in a distributed way, so as to pursue a global goal. This structure allows to different CIE collaborate in achieving objectives, that individually they cannot reach. This philosophy is based on the "Enterprise Networks" [22] through which a set of groups, institutions, or organizations interact, to obtain good results as much individual units in the network as a whole. It is precisely this approach that makes SCODA is based on the concept of Intelligent Community, as an organizational unit, with the features needed to carry out their development in these terms.

Table 1 Principles of SCODA

\begin{tabular}{ll}
\hline Principle & Description \\
\hline Standard & $\begin{array}{l}\text { Different Specialized Smart Communities have the same structure, } \\
\text { that is independent of the purpose to be achieved in the multi-agent } \\
\text { system to implement } \\
\text { This principle is based on human specialization, and in the Enterprise } \\
\text { Specialization } \\
\text { Intelligent Communities. } \\
\text { The multi-agent systems, within the context of architecture SCODA, } \\
\text { must be easy to implement. This is achieved because the structure of } \\
\text { Facility of Implementation } \\
\text { the Specialized Intelligent Communities is standard, being the } \\
\text { services they offer, which they have to be programmed. } \\
\text { Having in account that, within SCODA, each Specialized Intelligent } \\
\text { Community is adopted, as an independent multi-agent system, the } \\
\text { reuse of these Intelligent Communities Specialized has to be viable in } \\
\text { any SCODA based development. } \\
\text { The required services are not provided directly by agents of } \\
\text { Specialized Intelligent Communities. They are running in a } \\
\text { distributed way so that the computational load associated with } \\
\text { agents, decreases, and structure of the architecture has no variations. }\end{array}$ \\
Distributed Computation
\end{tabular}


SCODA is an architecture that can be implemented on any platform for multiagent systems that support BDI agents [23][24]. To carry out the development and implementation of this architecture is selected JADEX [25][26] because it is considered a reasoning engine and can be executed independently. SCODA architecture is based on five principles, through which seek greater efficiency of multi-agent systems developed with it.

SCODA is structured in a modular way, so that the agents, who compose it, can manage and coordinate the architecture and its functionality. It defines six basic modules: External Applications, Communications Protocol, Control, platform of agents, Specialized Intelligent Communities, and Services of the Communities. External applications are programs and users that use SCODA, requesting the services offered. The communication protocol is responsible for meeting the demands of external applications and request a response to the Specialized Intelligent Communities. The Control Module realises the follow-up of the coordination and functionality of the architecture, taking in account a fault tolerance policy in operation. The Specialized Intelligent Communities are the core of SCODA. Through by them are effective requests and responses, deliberately and of optimized form. The Community Services that running in a distributed way, is where find, the ability to processing each Specialized Intelligent Community. Finally, the agent platform, represents the environment where SCODA runs, and is composed of the agents that make up the architecture, and of the Specialized Intelligent Communities.

The agents that compose SCODA are deliberative agents BDI [23][24][27][28] and the services offered by CIE are managed by this type of agents. Another feature that is applied to all agents that make SCODA, is that being deliberative BDI agents, they can make use of reasoning mechanisms and learning techniques to perform the manage functionalities and coordination of them, depending on the particularities of the context where they run.

\section{$4 \quad$ Results}

To evaluate the system, we have the support of a company named Bahia Príncipe Congelados, which is specializing in the sale and distribution of frozen products. The services we have developed on SCODA, have been sales forecasting, inventory management and route optimization. The assessment raises a number of issues that affect both the architecture and in the result of the execution of the services associated with each community and its integration from SCODA. The system evaluation was performed for five months between 01/01/2012 and $31 / 05 / 2012$, during which the deployed multi-agent system has worked as proposed in this section, so that allowed us to gather the information necessary to perform an analysis of their performance and draw a number of conclusions.

Over these five months, the system shows an optimal performance, in terms of integration, since it has been found that there have been only sporadic errors that correspond to failures in services of the communities. These failures have been 
produced by unexpected errors in the server hosting the services of CIE, because taking account on the principle of distributed computing, services are hosted on a different server to the CIE.

The specialization of the CIE, allows them to be functional individually, and by themselves solve simple problems, for which, its associated services are qualified. Also, the union of the specialization of each one of CIE, emulating an enterprise network, will obtain the benefit of solving more complex problems so that each contributes its capabilities, being SCODA binding common framework and orchestrating them. This translates into the ability of the CIE implemented, to cooperate together and produce the result of the target system, taking account that these implementations can be used in other systems, independently of their targets.

In Table 2 are show the results of applying route optimization over SCODA. The benefits obtained are important, due to daily fuel saving, that multiplied by a large fleet of trucks, has a positive effect on the company. The subsystem of inventories management, and the prediction subsystem, they do not obtain a results as good as routes optimization.

Table 2 Results on Route Optimization

\begin{tabular}{llll}
\hline Route & Actual Km & Optimized Km & Improved Km \\
\hline Route A & 390,4 & 383,9 & 6,5 \\
Route B & 329,8 & 283,7 & 46,1 \\
Route C & 147,9 & 113,8 & 34,1 \\
Route D & 89,8 & 89,8 & 0 \\
Route E & 356,1 & 279,2 & 76,9 \\
\hline
\end{tabular}

\section{Conclusions}

The concept of community as a type of organizational unit joined to the concept of specialization, applied to multi-agent systems, have allowed to develop an architecture, named (SCODA), for developing, distributed multi-agent systems, regardless of the end that they pursue. The implementation of a system that can resolve logistics problems, has allowed us to test the operation of the architecture in a live environment, yielding promising results as much in, its integration as in terms of solving the given problems.

From these results it intends to continue working in this direction, and getting future developments in other areas.

Acknowledgments. This work has been partially supported by the MICINN project TIN 2009-13839-C03-03. 


\section{References}

1. Rashid, A., Moreira, A., Araujo, J.: Modularization and composition of aspectual requirements. In: 2nd International Conference on Aspect-Oriented Software Development, pp. 11-20 (2003)

2. Kiczales, G., Lamping, J., Mendhekar, A., Maeda, C., Lopes, C., Loingtier, J., Irwin, J.: Aspect-Oriented Programming. In: Akşit, M., Matsuoka, S. (eds.) ECOOP 1997. LNCS, vol. 1241, pp. 220-242. Springer, Heidelberg (1997)

3. Zambonelli, F., Jennings, N.R., Wooldridge, M.: Organizational abstractions for the analysis and design of multi-agent systems. In: Ciancarini, P., Wooldridge, M.J. (eds.) AOSE 2000. LNCS, vol. 1957, pp. 235-251. Springer, Heidelberg (2001)

4. Ferber, J., Gutknecht, O., Michel, F.: From agents to organizations: An organizational view of multi-agent systems. In: Giorgini, P., Müller, J.P., Odell, J.J. (eds.) AOSE 2003. LNCS, vol. 2935, pp. 214-230. Springer, Heidelberg (2004)

5. Hübner, J.F., Sichman, J.S., Boissier, O.: S-Moise ${ }^{+}$: A middleware for developing organised multi-agent systems. In: Boissier, O., Padget, J., Dignum, V., Lindemann, G., Matson, E., Ossowski, S., Sichman, J.S., Vázquez-Salceda, J. (eds.) ANIREM 2005 and OOOP 2005. LNCS (LNAI), vol. 3913, pp. 64-78. Springer, Heidelberg (2006)

6. Zambonelli, F., Jennings, N., Wooldridge, M.: Developing Multiagent Systems: The Gaia Methodology. ACM Transactions on Software Engineering and Methodology 12, 317-370 (2003)

7. Pavón, J., Gómez-Sanz, J.: Agent Oriented Software Engineering with INGENIAS. In: Mařík, V., Pěchouček, M., Müller, J. (eds.) CEEMAS 2003. LNCS (LNAI), vol. 2691, pp. 394-403. Springer, Heidelberg (2003)

8. Pavón, J., Gómez-Sanz, J.J., Fuentes, R.: The INGENIAS Methodology and Tools. In: Henderson-Sellers, B., Giorgini, P. (eds.) Agent Oriented Methodologies, pp. 236276. IDEA Group Publishing (2005)

9. Dignum, V., Dignum, F.: A landscape of agent systems for the real world. Technical Report 44-CS-2006-061. Institute of Information and Computing Sciences, Utrecht University (2006)

10. Corchado, J.M.: Agentes Software y Sistemas Multiagente. Pearson Education (2005), Pérez de la Cruz, J.L. (ed.)

11. Snyder, R., MacKenzie, D.: Cougaar Agent Communities. In: Proceedings of the 1st Open Cougaar Conference, New York City, pp. 143-148 (2004)

12. Nicklisch, H.: Cuestiones fundamentales de la Economía de Empresa, Stuttgart (1928)

13. Nicklisch, H.: La economía de empresa, Stuttgart (1932)

14. Larsen, B.: German organization and leadership theory-stable trends and flexible adaptation. Scandinavian Journal of Management 19(2003), 103-133 (2000)

15. Glinton, R., Paruchuri, P., Scerri, P., Sycara, K.: Self-Organized Criticality of Belief Propagation in Large Heterogeneous Teams. In: Hirsch, M.J., Pardalos, P.M., Murphey, R. (eds.) Dynamics of Information Systems: Theory and Applications. Springer, Berlin (2010)

16. Parachuri, P., Glinton, R., Sycara, K., Scerri, P.: Effect of humans on belief propagation in large heterogeneous teams. In: Hirsch, M.J., Pardalos, P.M., Murphey, R. (eds.) Dynamics of Information Systems: Theory and Applications. Springer, Berlin (2010) 
17. Theraulaz, G., Gervet, J., Semenoff, S.: Social regulation of foraging activities in polistes dominulus christ: a systemic approach to behavioural organization. Behaviour 116(1), 292-320 (1991)

18. Chai, L., Chen, J., Han, Z., Di, Z., Fan, Y.: Emergence of Specialization from Global Optimizing Evolution in a Multi-agent System. In: Shi, Y., van Albada, G.D., Dongarra, J., Sloot, P.M.A. (eds.) ICCS 2007, Part IV. LNCS, vol. 4490, pp. 98-105. Springer, Heidelberg (2007)

19. Okamoto, S., Scerri, P., Sycara, K.: The Impact of Vertical Specialization on Hierarchical Multi-Agent Systems. In: Proceedings of the Twenty-Third AAAI Conference on Artificial Intelligence (2008)

20. Mintzberg, H.: El diseño de las organizaciones eficientes. Ed. El Ateneo. Argentina (1989)

21. Román, J.A., Tapia, D.I., Corchado, J.M.: SCODA para el Desarrollo de Sistemas Multiagente. Revista Ibérica de Sistemas y Tecnologías de Información 8, 25-38 (2011)

22. Becerra, F.: Las redes empresariales y la dinámica de la empresa: aproximación teórica. INNOVAR. Revista de Ciencias Administrativas y Sociales (en línea), 18 (2008), Disponible en

http://redalyc.uaemex.mx/src/inicio/

ArtPdfRed.jsp?iCve $=81803203$

23. Bratman, M.E., Israel, D., Pollack, M.: Plans and resource-bounded practical reasoning. Computational Intelligence 4, 349-355 (1988)

24. Rao, A.S., Georgeff, M.P.: BDI Agents from Theory to Practice. In: Proceedings of the First International Conference on Multi-Agents Systems (ICMAS 1995), pp. 312-319 (1995)

25. Pokahr, A., Braubach, L., Lamersdorf, W.: Jadex: Implementing a BDI-Infrastructure for JADE Agents. In EXP - in search of innovation (Special Issue on JADE), 76-85 (2003)

26. Pokahr, A., Braubach, L., Walczak, A., Lamersdorf, W.: Jadex - Engineering GoalOriented Agents. In: Developing Multi-Agent Systems with JADE, pp. 254-258. Wiley \& Sons (2007)

27. Koster, A., Schorlemmer, M., Sabater, J.: Opening the black box of trust: reasoning about trust models in a BDI agent. Journal of Logic and Computation (2012)

28. van Oijen, J., Dignum, F.: Towards a Design Approach for Integrating BDI Agents in Virtual Environments. In: Vilhjálmsson, H.H., Kopp, S., Marsella, S., Thórisson, K.R. (eds.) IVA 2011. LNCS, vol. 6895, pp. 462-463. Springer, Heidelberg (2011)

29. Posland, S.: Specifying protocols for multi-agent systems interaction. ACM Trans. Auton. Adapt. Systems 2(4), 15 (2007) 